\title{
Review of the Psychological Impact of COVID-19 Pandemic on Healthcare Workers in Saudi Arabia
}

\author{
Ahmed S Bahamdan \\ Family and Community Medicine \\ Department, College of Medicine, Imam \\ Abdulrahman Bin Faisal University, \\ Dammam, Saudi Arabia
}

\begin{abstract}
Purpose: The Covid-19 pandemic has significantly burdened healthcare systems worldwide and substantially affected the psychological state. The objective of this narrative review was to summarize the psychological outcome of the "Coronavirus (COVID-19) pandemic" on healthcare workers in kingdom of Saudi Arabia to assess their mental health outcome that can aid in development of guidelines and psychological interventions that can improve healthcare workers' quality of life, work and decision-making capability toward patient treatment during the pandemic.
\end{abstract}

Materials and Methods: A comprehensive research was done to overview current available literature on psychological and mental health issues observed among healthcare workers "HCW" in Saudi Arabia. The search included all articles published since the beginning of the pandemic from January 2020 till February 2021 relevant to the subject of the review. In this review, a total of 10 primary research articles were included following a cross-sectional survey method to analyze the impact of various psychological variables.

Results: Anxiety symptoms were reported by between $33.3 \%$ and $68.5 \%$ of HCWs. Between $27.9 \%$ and $55.2 \%$ of HCWs reported depressive symptoms. HCWs reported anxiety, depression, sleep disturbances and distress with a range comprised between $27.9 \%$ and $68.5 \%$.

Conclusion: The Covid-19 pandemic has certainly led to multifaceted and vigorous psychological and mental impact on healthcare providers, it is now both an opportunity and challenge to design further studies that can lead to development of guidelines in Saudi Arabia and worldwide to improve mental health infrastructure that strengthen patient oriented treatment of care plan during this pandemic.

Keywords: anxiety, depression, stress, insomnia

\section{Introduction}

In the year 2020, the World Health Organization (WHO) declared the coronavirus outbreak as a global emergency. A year has been passed to this pandemic and the prevalence of psychological issues is rising day by day as the pandemic progresses. It has come across as an unprecedented dispute for healthcare systems. Generally, pandemics require immediate and efficient response from healthcare systems, with many healthcare workers (HCWs), either involved directly such as doctors and nurses or indirectly like laboratory and radiology technicians providing patients with care, fighting at the frontline and addressing challenges that threaten healthcare systems. ${ }^{1}$

History has showed that dynamic stress is experienced by HCWs during outbreaks. In a Chinese study among HCWs during the Ebola outbreak, extreme anxiety, depression, somatization and obsession-compulsions were reported. ${ }^{2}$
Correspondence: Ahmed S Bahamdan Family and Community Medicine Department, College of Medicine, Imam Abdulrahman Bin Faisal University, Dammam, 3422I - 4237, Saudi Arabia

Tel +966546004777

Fax +966 I33330220

Email bahamdan.as@gmail.com 
Also, in a study in Saudi Arabia, almost two thirds of HCWs reported feeling at risk of getting Middle East respiratory syndrome coronavirus (MERS-CoV) infection and felt unsafe working during the MERS outbreak. ${ }^{3}$

In Saudi Arabia, since the beginning of the pandemic until the 1st of December, 2020, there were 347,157 confirmed (Covid-19) cases, with 5907 deaths. ${ }^{4}$ Until this date, there is no cure or approved vaccine for Covid-19. On top of that, an increase in depression, stress and anxiety symptoms has been reported in the general population and, especially, in HCWs. Increased working duties and the need to make difficult decisions on prioritizing care of patients may have strong effects on HCWs' physical and mental health. Their resilience can be further affected by isolation and loss of social support, risk of infections or infecting family and friends as well as desperate, often unsettling changes in their work responsibilities. ${ }^{5}$

Thus, HCWs especially frontline staff face critical conditions which increase their risk of disturbing their mental health after dealing with unfavorable situations that can range from psychological distress to psychological symptoms more than the general population. ${ }^{6,7}$ Fighting against a new virus with an unknown nature for a prolonged time is a crisis that affects the sustainability of healthcare systems. Maintaining the maximum possible care provided fully relies on protecting the health of those responding to such crisis. Yet, the published findings of psychological distress among HCWs might indicate that healthcare systems are currently unable to effectively protect the helpers. ${ }^{8}$

Psychological impacts and fear or hesitancy in HCWs may affect the efficiency, and quality of work as well as willingness to report to work which can have a dramatic effect on healthcare systems especially during pandemics. ${ }^{9}$ Reported physicians committing suicide with the psychological impacts reported worldwide, led me to review the current literature of this pandemic on HCWs in Saudi Arabia and the latest recommendation regarding their mental health. ${ }^{10,11}$

The objective of this narrative review is to summarize the psychological outcome of the "Coronavirus (Covid-19) pandemic" on healthcare workers in kingdom of Saudi Arabia to assess their mental health outcome that can aid in development of guidelines and psychological interventions that can improve healthcare workers' quality of life, work and decision-making capability toward patient treatment during pandemic.

\section{Materials and Methods}

A comprehensive research was done to overview current available primary literature on psychological and mental health issues observed among healthcare workers " $\mathrm{HCW}$ " in Saudi Arabia. Literature search was conducted in "PubMed (MEDLINE)", "ProQuest" and "Web of Science" database. Data were retrieved using the following search terms ("medical staff" OR "healthcare" OR "healthcare professionals" OR "Physicians") AND ("coronavirus" OR "COVID-19”) AND ("anxiety” OR "depression" OR "insomnia" OR "psychological" OR "mental health" OR "Stress") AND (in "Saudi Arabia" OR "KSA") in our search engines.

Our search included all primary articles published in peer reviewed journals since the beginning of pandemic from January 2020 till February 2021 relevant to the subject of the review catering original research. Review articles, correspondence, case reports and non-English articles were excluded. A final total of 89 citations were identified through search terms collectively from all databases. During initial screening 19 citations were removed as duplicate. Out of 70 after removing duplicate articles, 45 articles including review articles, correspondence and case reports were removed. Out of 25, a further 15 articles were removed as they were not conducted on $\mathrm{HCW}$ and assessed knowledge, attitude and practices. A total of final 10 full texts, primary articles were included. Results of the potential 10 articles were summarized in the form of Table 1 and health variables including anxiety, stress, sleep and depression are assessed in all studies (Figure 1).

\section{Results}

In this review, a total of 10 citations were included following a cross-sectional survey method to analyze the impact of various psychological variables including anxiety, stress, sleep and depression among Saudi healthcare workers during the Covid-19 pandemic. All 10 studies were evaluated to assess psychological symptom prevalence in both males and females. The psychological symptom prevalence was profoundly observed in females (6 studies) in comparison to males (4 studies) (Figure 2). The most frequently used assessment tools were General Anxiety Disorder (GAD-7) and Patient's Health Questionnaire (PHQ-9) respectively. However, other tools including Depression Anxiety Stress Scale-21 (DASS-21) and the psychological distress using the COVID-19 Peritraumatic Distress Index (CPDI) were also used. 


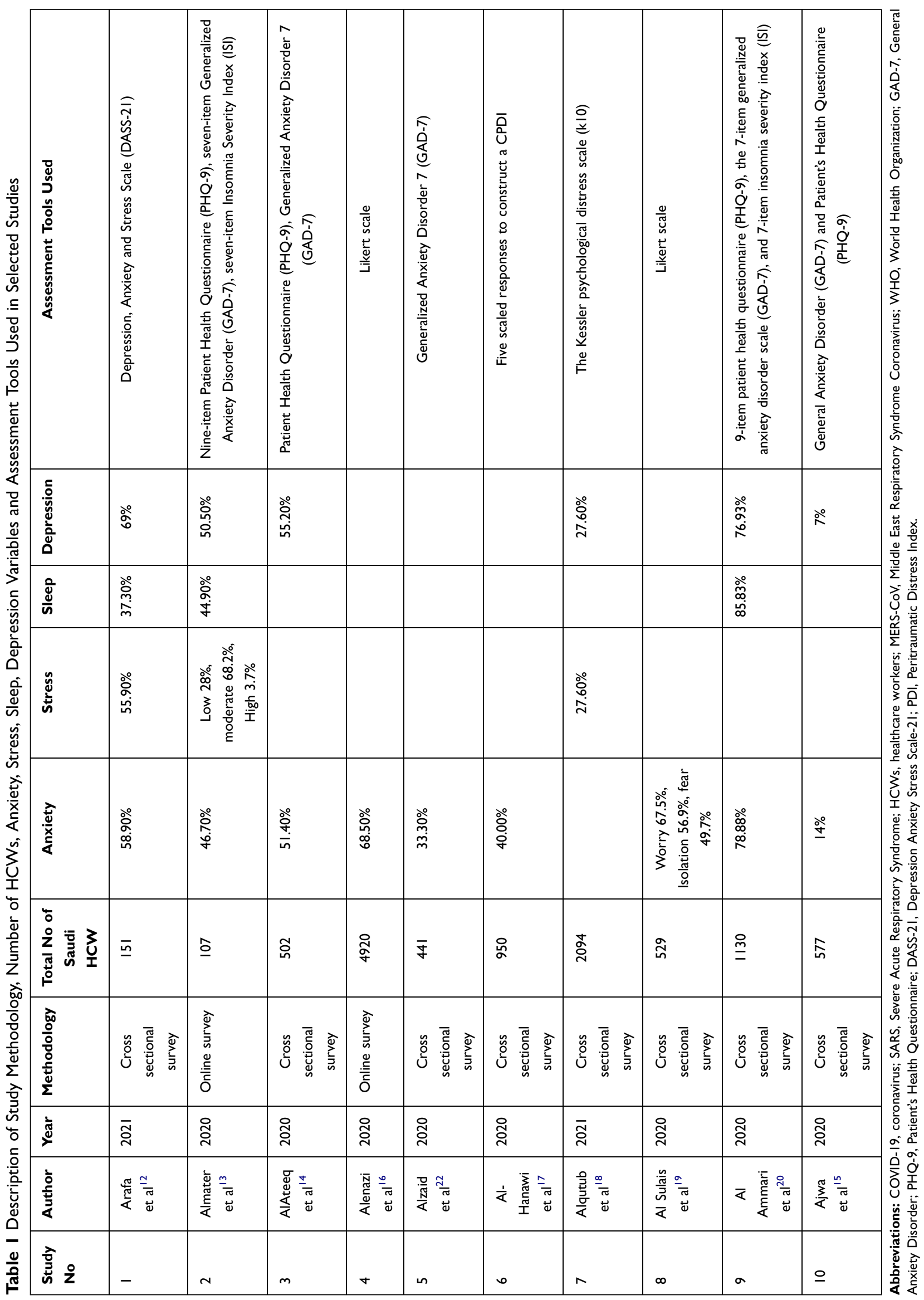



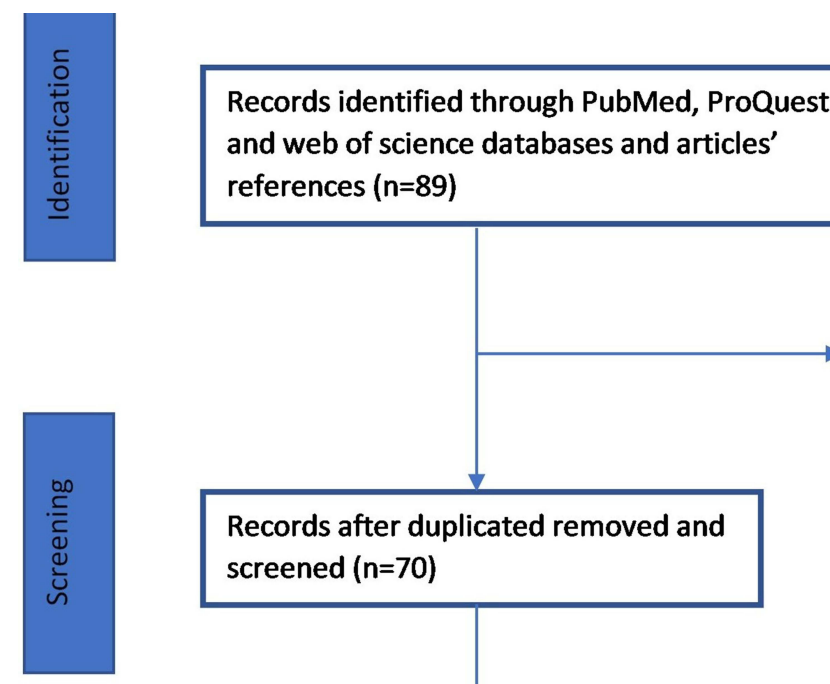

\section{Records after duplicated removed and}

screened $(n=70)$

Duplicates removed

$(n=19)$

Reviews, editorials,

different subjects $(n=45)$

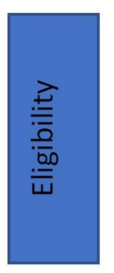

Full-text articles assessed for eligibility $(n=25)$
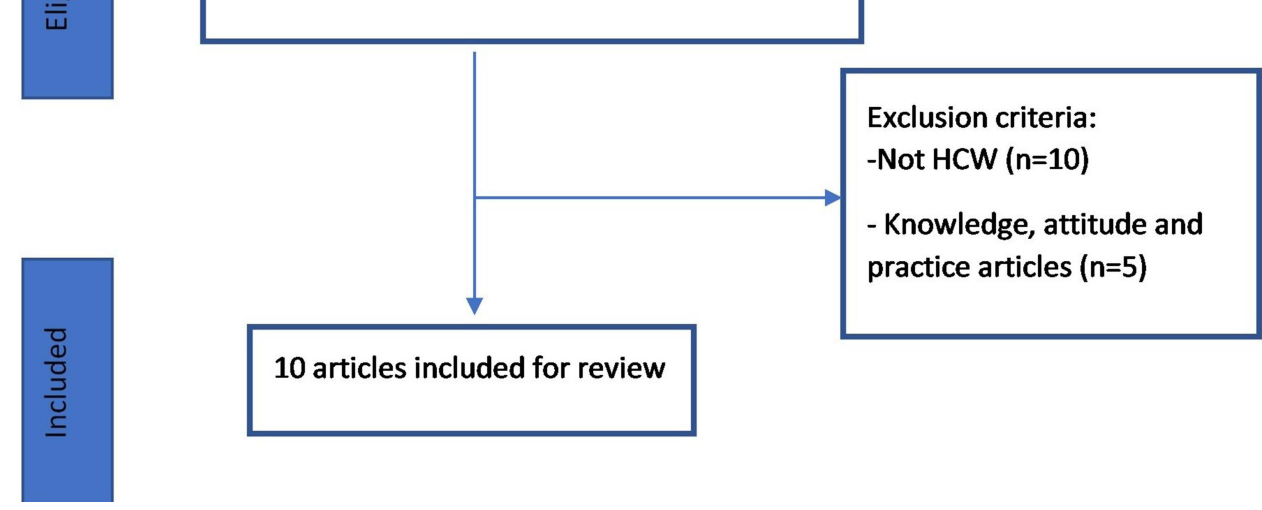

10 articles included for review

Figure I Eligibility criteria for inclusion and exclusion of studies in the review.

In this study, Arafa et al ${ }^{12}$ reported highest progression of depression symptoms $69 \%$ in comparison to anxiety $58.90 \%$, stress $55.9 \%$ and inadequate sleep $37.3 \%$ respectively among healthcare providers. He further graded depression as $29.6 \%$ with severe depressive symptoms and $39.4 \%$ with mild to moderate depression. Another study by Almater et $\mathrm{al}^{13}$ had further explained grading of stress among healthcare practitioners with stress low levels (28\%), moderate levels $(68.2 \%)$ and high levels (3.7\%).

Two studies by AlAteeq et al ${ }^{14}$ and Nancy Ajwa et al ${ }^{15}$ explained both anxiety and depression among healthcare professionals and observed more depressive and anxious symptoms were observed in professionals deputed in medical wards as compared to dental ward. The largest study was done in a nation-level cross-sectional study of participants from all the 13 administrative regions in Saudi Arabia with a sample of 4920 HCWs. They divided them into three groups according to anxiety level on the Dispositional cancer worry scale, 1552 (31.5\%) low, 1778 (36.1\%) medium, and 1590 (32.3\%) high anxiety. ${ }^{16}$

In Al-Hanawi et $\mathrm{al}^{17}$ the study sample was 950 and (28.9\%) showed normal, (33.7\%) mild and (39.9\%) severe distress using the COVID-19 Peritraumatic Distress Index which is a self-reported questionnaire that was originally used by a study in China to assess peritraumatic psychological distress during the pandemic. Another study by Alqutub et $\mathrm{al}^{18}$ used the Kessler psychological distress scale $(\mathrm{k} 10)$ question, and found that $27.6 \%$ healthcare workers were in psychological distress. 


\section{Gender wise Variables Prevalence}

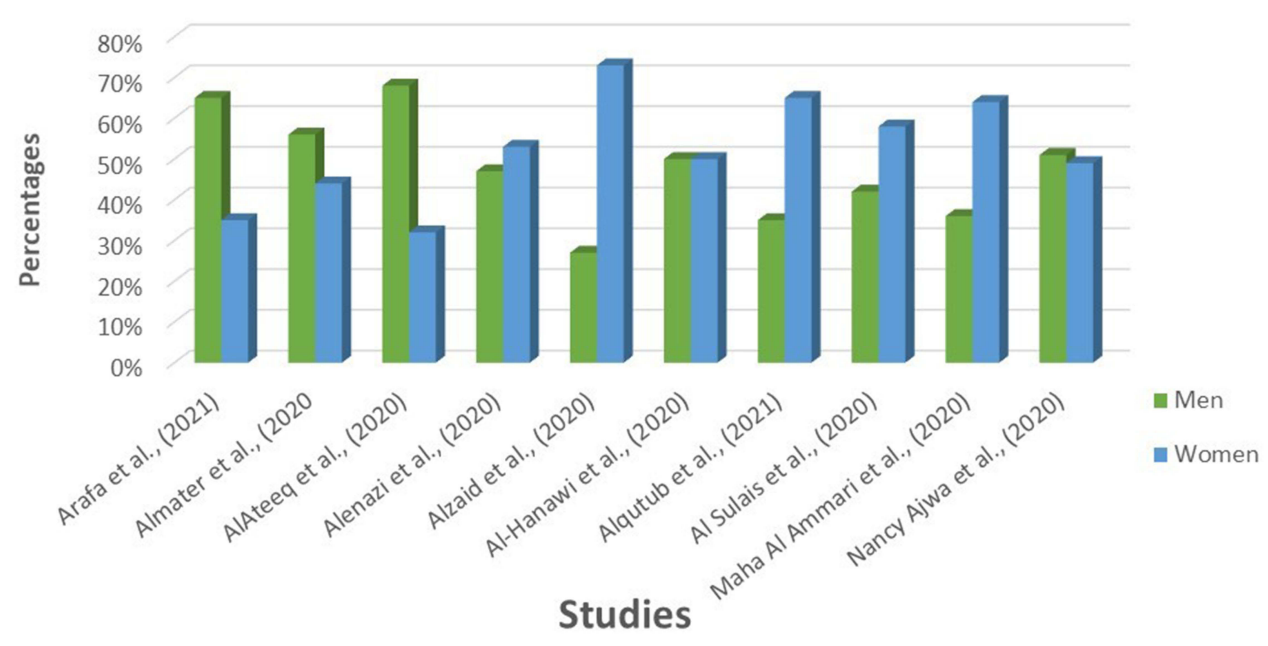

Figure 2 Gender-wise psychological variable prevalence.

Al Sulais et $\mathrm{al}^{19}$ used a questionnaire designed by Reynolds et al and observed that the most common feelings reported by the physicians during the pandemic were: worry (67.5\%), isolation (56.9\%) and fear (49.7\%) through using a Likert scale. Similarly, Al Ammari et $\mathrm{al}^{20}$ with participation of 1130 participants from the healthcare sector showed $78.88 \%$ symptoms of work induced psychological anxiety, $85.83 \%$ disturbance in sleep cycle or inability to sleep properly which ultimately led to $76.93 \%$ symptoms of depression.

\section{Discussion}

This review provided the psychological impact of the COVID-19 pandemic on healthcare workers in Saudi Arabia. Overall, $60 \%$ of the studies reviewed in this article found out that more than $50 \%$ of the healthcare workers had psychological impact in terms of depression, anxiety and stress during the global emergency.

The studies included in our review reflected females as predominant participants in 6 studies (64.40-72.80\%). Similarly, Lai et $\mathrm{al}^{7}$ in their study reflected professional women showed moderate to severe depression, anxiety and stress. Arafa et $\mathrm{al}^{12}$ in their study stated that mostly allied healthcare providers missed family emotional support and indulged in watching covid news that increased likelihood of stress, disturbed sleep, anxiety and depression among patients. In correlation to it, to improve overall $\mathrm{HCW}$ mental health status, Banerjee ${ }^{21}$ emphasized on psychiatrist significance in terms of education on pandemic psychological impact on public, motivation to develop and adopt strategies to prevent the spread of novel virus through basic practices and its integration in available healthcare setups. It also suggested how to adopt a problem solving approach and empowering both caregivers and patients through provision of quality mental healthcare services to care providers.

In a study conducted in China, prevalence distress symptoms were highest $(71.5 \%)$, followed by depression $(50.4 \%)$, anxiety (44.6\%) and insomnia (34.0\%) respectively. ${ }^{7}$ Overall, our studies have showed more prevalence of depression and anxiety in comparison to stress and insomnia symptoms. In another study by Almater et $\mathrm{al}^{13}$ impact of Covid-19 was observed in ophthalmologist setup. During earlier pandemics healthcare professionals felt unprotected and lethargic, stressed during working in high-risk areas, however no significant previous work was identified regarding safety and psychological counseling of ophthalmologists during exposure with patients during work. Ophthalmologists are also at higher risk of virus transmission because of increased exposure to droplet or contact routes while performing slit lamp examination.

Longitudinal research studies are important to follow up on HCWs' mental health and develop intervention strategies that are evidence-based. Routine mental health screening is encouraged especially during pandemics. ${ }^{16,17,22}$ The World Health Organization's mental health department published strategies to decrease the negative psychological impact of the COVID-19 pandemic to the general population and HCWs. A well-balanced lifestyle including regular exercise, 
healthy diet and enough sleep is advised. Staying connected with families and friends is also encouraged with the different virtual applications. ${ }^{23}$

This study provided the review of merely 10 original articles as limited primary data were available relevant to our objective. We could not apply the quality assessment tool in this review yet we performed a detailed assessment of every included article. Regarding the type of the study, as this pandemic was started in 2020 and a short time span has passed, primary data were scarce and most of the studies were cross sectional and none of the longitudinal studies could be reviewed. The response variability might occur as studies were conducted in different medical institutes and different stress, anxiety and depression scales were used.

\section{Conclusion}

The COVID-19 pandemic has certainly led multifaceted and vigorous psychological and mental impact on healthcare providers, it is now both an opportunity and challenge to design further studies that can lead to development of guidelines in Saudi Arabia and worldwide to improve mental health infrastructure that strengthen patient oriented treatment of care plan during this pandemic through development of mental health interventions that can overall improve productivity of healthcare provider.

\section{Acknowledgments}

The author is thankful to all the associated personnel, who contributed to this study by any means.

\section{Disclosure}

The author reports no conflicts of interest in this work.

\section{References}

1. Preti E, Di Mattei V, Perego G, et al. The psychological impact of epidemic and pandemic outbreaks on healthcare workers: rapid review of the evidence. Curr Psychiatry Rep. 2020;22:1-22. doi:10.1007/ s11920-020-01166-Z

2. Ji D, Ji YJ, Duan XZ, et al. Prevalence of psychological symptoms among Ebola survivors and healthcare workers during the 2014-2015 Ebola outbreak in Sierra Leone: a cross-sectional study. Oncotarget. 2017;8:12784. doi:10.18632/oncotarget.14498

3. Abolfotouh MA, AlQarni AA, Al-Ghamdi SM, Salam M, Al-Assiri $\mathrm{MH}$, Balkhy HH. An assessment of the level of concern among hospital-based health-care workers regarding MERS outbreaks in Saudi Arabia. BMC Infect Dis. 2017;17:1. doi:10.1186/s12879-0162096-8

4. Saudi Ministry of Health; 2020. Available from: https://covid19.moh. gov.sa/. Accessed December 1, 2020.
5. Pappa S, Ntella V, Giannakas T, Giannakoulis VG, Papoutsi E, Katsaounou P. Prevalence of depression, anxiety, and insomnia among healthcare workers during the COVID-19 pandemic: a systematic review and meta-analysis. Brain Behav Immun. 2020;88:901-907. doi:10.1016/j.bbi.2020.05.026

6. Zhou Y, Wang W, Sun Y, et al. The prevalence and risk factors of psychological disturbances of frontline medical staff in China under the COVID-19 epidemic: workload should be concerned. $J$ Affect Disord. 2020;277:510-514. doi:10.1016/j.jad.2020.08.059

7. Lai J, Ma S, Wang Y, et al. Factors associated with mental health outcomes among health care workers exposed to coronavirus disease 2019. JAMA Netw Open. 2020;3:e203976. doi:10.1001/jamanetworkopen.2020.3976

8. Muller RA, Stensland RS, van de Velde RS. The mental health impact of the covid-19 pandemic on healthcare workers, and interventions to help them: a rapid systematic review. Psychiatry Res. 2020;293:113441. doi:10.1016/j.psychres.2020.113441

9. Almaghrabi RH, Huda A, Al HW, Albaadani MM. Healthcare workers experience in dealing with Coronavirus (COVID-19) pandemic. Saudi Med J. 2020;41:657-660. doi:10.15537/smj.2020.6.25101

10. NBC New York. NYC emergency room doctor dies by suicide after treating COVID-19 patients; 2020. Available from: https://www.nbcne wyork.com/news/coronavirus/nyc-emergency-room-doctordies-fromsuicide-after-treating-covid-19-patients/2391978/. Accessed December 1, 2020.

11. NST Online. French doctor commits suicide after Covid-19 diagnosis. New Straits Times; 2020. Available from: https://www. nst.com.my/world/world/2020/04/581620/french-doctor-commitssuicide-after-covid-19-diagnosis. Accessed December 1, 2020.

12. Arafa A, Mohammed Z, Mahmoud O, Elshazley M, Ewis A. Depressed, anxious, and stressed: what have healthcare workers on the frontlines in Egypt and Saudi Arabia experienced during the COVID-19 pandemic? J Affect Disord. 2021;278:365-371. doi:10.1016/j.jad.2020.09.080

13. Almater AI, Tobaigy MF, Younis AS, Alaqeel MK, Abouammoh MA. Effect of 2019 coronavirus pandemic on ophthalmologists practicing in Saudi Arabia: a psychological health assessment. Middle East Afr $J$ Ophthalmol. 2020;27:79. doi:10.4103/meajo.meajo_220_20

14. AlAteeq DA, Aljhani S, Althiyabi I, Majzoub S. Mental health among healthcare providers during coronavirus disease (COVID-19) outbreak in Saudi Arabia. $J$ Infect Public Health. 2020;13:1432-1437. doi:10.1016/j.jiph.2020.08.013

15. Ajwa N, Al Rafee A, Al Rafie H, et al. Psychological status assessment of medical and dental staff during the covid-19 outbreak in Saudi Arabia. Med Sci. 2020;24:4790-4797.

16. Alenazi TH, BinDhim NF, Alenazi MH, et al. Prevalence and predictors of anxiety among healthcare workers in Saudi Arabia during the COVID-19 pandemic. $J$ Infect Public Health. 2020;13:1645-1651. doi:10.1016/j.jiph.2020.09.001

17. Al-Hanawi MK, Mwale ML, Alshareef N, et al. Psychological distress amongst health workers and the general public during the COVID-19 pandemic in Saudi Arabia. Risk Manag Healthc Policy. 2020;13:733. doi:10.2147/rmhp.s264037

18. Alqutub S, Mahmoud M, Baksh T. Psychological impact of COVID-19 on frontline healthcare workers in Saudi Arabia. Cureus. 2021;13(5):e15300.

19. Al Sulais E, Mosli M, AlAmeel T. The psychological impact of COVID-19 pandemic on physicians in Saudi Arabia: a cross-sectional study. Saudi J Gastroenterol. 2020;26:249. doi:10.4103/sjg.sjg_174_20

20. Al Ammari M, Sultana K, Thomas A, Al Swaidan L, Al Harthi N. Mental health outcomes amongst health care workers during COVID 19 pandemic in Saudi Arabia. Front Psychiatry. 2020;11. doi: $10.3389 /$ fpsyt.2020.619540.

21. Banerjee D. The COVID-19 outbreak: crucial role the psychiatrists can play. Asian J Psychiatr. 2020;50:102014. doi:10.1016/j. ajp.2020.102014 
22. Alzaid EH, Alsaad SS, Alshakhis N, Albagshi D, Albesher R, Aloqaili M. Prevalence of COVID-19-related anxiety among healthcare workers: a cross-sectional study. J Fam Med Prim Care. 2020;9:4904. doi:10.4103/jfmpc.jfmpc_674_20
23. Who.int; 2020. Available from: https://www.who.int/docs/default-source /coronaviruse/mental-health-considerations. Accessed December 1, 2020.

\section{Publish your work in this journal}

Risk Management and Healthcare Policy is an international, peerreviewed, open access journal focusing on all aspects of public health, policy, and preventative measures to promote good health and improve morbidity and mortality in the population. The journa welcomes submitted papers covering original research, basic science, clinical \& epidemiological studies, reviews and evaluations, guidelines, expert opinion and commentary, case reports and extended reports. The manuscript management system is completely online and includes a very quick and fair peer-review system, which is all easy to use. Visit http://www.dovepress.com/testimonials.php to read real quotes from published authors.

Submit your manuscript here: https://www.dovepress.com/risk-management-and-healthcare-policy-journal 